\title{
The prognostic significance of lymphovascular invasion in patients with resectable gastric cancer: a large retrospective study from Southern China
}

\author{
Peng $\mathrm{Li}^{1,2+}$, Hao-Qiang He ${ }^{1,3+}$, Chong-Mei Zhu ${ }^{1,2}$, Yi-Hong Ling ${ }^{1,2}$, Wan-Ming Hu ${ }^{1,2}$, Xin-Ke Zhang ${ }^{1,2}$, \\ Rong-Zhen Luo ${ }^{1,2}$, Jing-Ping Yun ${ }^{1,2}$, Dan Xie ${ }^{1}$, Yuan-Fang Li $i^{1,4^{*}}$ and Mu-Yan Cai ${ }^{1,2^{*}}$
}

\begin{abstract}
Background: The focus of this study was to assess the impact of lymphovascular invasion (LVI) on both the recurrence of cancer and the long-term survival of Chinese patients with resectable gastric cancer (GC).

Methods: A retrospective analysis of the clinicopathological data for 1148 GC patients who had undergone gastrectomy with regional lymphadenectomy was performed. The primary objective was to assess the correlation between LVI and post-surgery outcomes for each patient. This was done by routine H \& E staining for LVI on patients' disease-free survival (DFS) and disease-specific survival (DSS).

Results: LVI was detected in 404 (35.2\%) of the 1148 GC patients. The presence of LVI was significantly correlated with the level of CA19-9, the tumor size, the Lauren classification, tumor differentiation, gastric wall invasive depth, lymph node involvement, distant metastasis and an advanced TNM stage. There was a lower DFS and DSS in the patients with LVI as compared to the patients without LVI. A multivariate analysis also identified LVI as an independent prognostic factor of both DSS and DFS.
\end{abstract}

Conclusions: The presence of LVI is a risk factor for the recurrence of cancer and an independent indicator of a poor outcome in GC patients following surgery. The LVI status should be taken into consideration when determining the best approach for the treatment of the individual.

Keywords: Lymphovascular invasion, Prognosis, Gastric cancer

\section{Background}

Gastric cancer (GC) continues to be a major challenge in the health care community worldwide especially in East Asian countries; such as China, South Korea and Japan $[1,2]$. Despite the advances in medical treatments, gastrectomy with regional lymphadenectomy remains the primary treatment for patients with resectable GC and has a five-year overall survival (OS) rate of approximately $20-30 \%$. The low OS rate is due to the high frequency in the post-surgery recurrence of cancer $[3,4]$.

\footnotetext{
* Correspondence: liyuanf@sysucc.org.cn; caimuyan@hotmail.com ${ }^{\dagger}$ Equal contributors

${ }^{1}$ State Key Laboratory of Oncology in South China, Collaborative Innovation Center for Cancer Medicine, Sun Yat-sen University Cancer Center, Guangzhou, China

Full list of author information is available at the end of the article
}

The most reliable indication of the prognosis following surgery can be provided through the assessment of the GC using the International Union Against Cancer/American Joint Committee on Cancer (UICC/AJCC) TNM staging guidelines [5]. However, it has been noted that many patients that have been determined to have the same UICC/ AJCC TNM stage have heterogeneous survival rates. Therefore, there has been an increased focus on determining other prognostic indicators that will aid in the identification of GC patients with a higher risk for the recurrence of their cancer and who may be candidates for other adjuvant therapies.

The major factor contributing to the recurrence of cancer and mortality is thought to be the systemic dissemination of cancer cells. Lymphovascular invasion 
(LVI), also referred to as blood vessel and/or lymphatic invasion, is the presence of tumor cells within the lumen of the blood and/or lymphatics; the process of which leads to circulating tumor cells. The presence of LVI is a common pathological finding in a variety of human cancers and has been shown to be associated with a high recurrence rate and poor prognosis in patients with breast cancer, colorectal cancer, non-small cell lung cancer and clear cell renal cell carcinoma [6-10]. The combination of traditional TNM staging with an assessment for LVI could lead to a more accurate indication of the patient's prognosis [11].

Previous studies have investigated the prognostic significance of LVI in relation to GC in small selected cohorts. The results of these studies indicated that the presence of LVI, either in the blood or lymphatics, correlated with tumor recurrence and a low survival rate that appeared to be independent of lymph node status [8,12-18]. The prognostic value of an LVI assessment in $\mathrm{GC}$ remains controversial due to the small number of participants in the study. To address this issue, a large retrospective study of GC patients who had undergone surgery in Southern China was designed and carried out to thoroughly investigate the correlation between LVI and cancer-recurrence/ long-term survival.

\section{Methods}

\section{Patient selection}

Of the 3321 GC patients that had undergone surgery in Sun Yat-sen University Cancer Center (Guangzhou, China) between May of 1996 and June of 2009, 1148 (34.6\%) were selected because they had a gastrectomy with lymphoadenectomy. This was determined using the archives of the Department of Pathology based upon the following criteria: (1) a histologically confirmed primary gastric adenocarcinoma; (2) no neoadjuvant treatment before operation; (3) complete resection of the tumor; (4) resection margins were negative; (5) detailed and complete follow-up data.

Variables included the gender of the patient (female and male), age at the time of surgery $(<60$ and $\geq 60$ years), the level of preoperative serum carcinoembryonic antigen (CEA; elevated and normal), levels of the carbohydrate antigen (CA19-9; elevated and normal), approximate tumor size $(\leq 5$ and $>5 \mathrm{~cm}$ ), tumor differentiation (well, moderate and poor), Lauren classification (intestinal, mixed and diffuse), infiltration depth (T1, T2, T3 and T4), lymph node status (N0, N1, N2 and N3), distant metastasis (absent and presence), TNM stage (I, II, III and IV), LVI (absent and presence) and recurrence. Detailed information is given in Table 1 . The immunoradiometric method was used to measure the serum concentrations of both CEA and CA19-9. The cut-off values for CEA and CA19-9 were $5.0 \mathrm{ng} / \mathrm{ml}$ and $35.0 \mathrm{U} / \mathrm{ml}$;
Table 1 Correlation between lymphovascular invasion and clinicopathologic characteristics in gastric carcinoma

\begin{tabular}{|c|c|c|c|c|}
\hline \multirow[t]{2}{*}{ Variables } & \multicolumn{4}{|c|}{ Lymphovascular invasion } \\
\hline & All cases & Absence & Presence & $P$ value ${ }^{*}$ \\
\hline Sex & & & & 0.955 \\
\hline Female & 354 & $229(64.7 \%)$ & 125 (35.3\%) & \\
\hline Male & 794 & $515(64.9 \%)$ & 279 (35.1\%) & \\
\hline Age at diagnosis (years) & & & & 0.458 \\
\hline$<60$ & 574 & $378(65.9 \%)$ & 196 (34.1\%) & \\
\hline$\geq 60$ & 574 & $366(63.8 \%)$ & $208(36.2 \%)$ & \\
\hline $\mathrm{CEA}^{\dagger}$ & & & & 0.665 \\
\hline Normal & 791 & $525(66.4 \%)$ & $266(33.6 \%)$ & \\
\hline Elevated & 157 & $107(68.2 \%)$ & $50(31.8 \%)$ & \\
\hline CA19-9 & & & & 0.004 \\
\hline Normal & 707 & $487(68.9 \%)$ & $220(31.1 \%)$ & \\
\hline Elevated & 195 & $113(57.9 \%)$ & $82(42.1 \%)$ & \\
\hline Size (diameter), cm & & & & $<0.0001$ \\
\hline$\leq 5$ & 697 & 487 (69.9\%) & $210(30.1 \%)$ & \\
\hline$>5$ & 451 & $257(57.0 \%)$ & 194 (43.0\%) & \\
\hline Lauren classification & & & & 0.004 \\
\hline Diffuse & 585 & $356(60.9 \%)$ & 229 (39.1\%) & \\
\hline Mixed/ Intestinal & 563 & $388(68.9 \%)$ & 175 (31.1\%) & \\
\hline Differentiation & & & & $<0.0001$ \\
\hline Well/moderate & 435 & $331(76.1 \%)$ & 104 (23.9\%) & \\
\hline Poor/undifferentiated & 713 & $413(57.9 \%)$ & $300(42.1 \%)$ & \\
\hline Gastric wall invasion & & & & $<0.0001$ \\
\hline $\mathrm{T} 1 / \mathrm{T} 2$ & 150 & $140(93.3 \%)$ & $10(6.7 \%)$ & \\
\hline $\mathrm{T} 3 / \mathrm{T} 4$ & 998 & $604(60.5 \%)$ & 394 (39.5\%) & \\
\hline Nodal metastasis & & & & $<0.0001$ \\
\hline No & 377 & $322(85.4 \%)$ & $55(14.6 \%)$ & \\
\hline N1-N3 & 771 & $422(54.7 \%)$ & 349 (45.3\%) & \\
\hline Distant metastasis & & & & $<0.0001$ \\
\hline MO & 1003 & $679(67.7 \%)$ & $324(32.4 \%)$ & \\
\hline M1 & 145 & $65(44.8 \%)$ & $80(55.2 \%)$ & \\
\hline TNM stage & & & & $<0.0001$ \\
\hline$|/| \mid$ & 486 & 409 (84.2\%) & $77(15.8 \%)$ & \\
\hline III/ IV & 662 & $335(50.6 \%)$ & 327 (49.4\%) & \\
\hline
\end{tabular}

${ }^{*}$ Chi-square test; ${ }^{\dagger}$ Preoperative serum CEA was measured in 948 patients; †Preoperative serum CA19-9 was measured in 902 patients; CEA indicates carcinoembryonic antigen; CA19-9 indicates carbohydrate antigen 19-9.

serum concentrations found to be above these respective cut-off values were defined as elevated. In the first year post surgery patients were followed up every three months; the following two years they were seen every six months and annually thereafter. A complete history and physical examination, gastroscopy, gastrointestinal barium examination, CT and MRI was done in order to assess tumor recurrence; which was defined as local recurrence 
or metastasis. The disease-free survival (DFS) was defined as the time interval post-surgery until recurrence/metastasis or death from gastric cancer (GC), whichever came first. The disease-specific survival (DSS) was defined as the time interval post-surgery until the date of death resulting from GC or the date of the last follow-up exam. This study was approved by the Institute Research Medical Ethics Committee of Sun Yat-sen University Cancer Center. All patient information was hidden to reviewers. No informed consent, written or verbal, was obtained for the retrospective use of the tissue specimens from the patients in this study, however, since most were deceased approval was deemed unnecessary by the Ethics Committee and the need for consent was waived.

\section{Pathological evaluation}

Standard pathological procedures were followed in the processing of all surgical specimens. $\mathrm{H} \&$ E-stained slides of the primary tumors and regional lymph nodes were independently examined by two pathologists; both of whom had no prior knowledge of the clinical parameters of the patient. Discrepancies were resolved through the simultaneous re-examination of the slides using a double-headed microscope by both pathologists. For each tumor, there were at least three slides available for pathological evaluation. The WHO Classification of Tumours of the Digestive System (2010 version) was used to determine tumor differentiation. The depth of tumor infiltration, the lymph node status and the tumor stage was determined utilizing the UICC/AJCC TNM (tumornode-metastasis) Classification System (2010 version). LVI was defined as the invasion of vessel walls by tumor cells and/or the presence of tumor emboli within an endothelial-lined space; with no distinction between vascular and lymphatic vessels [8]. The following criterion was used to identify the lumen of blood and/or lymph vessels: (i) lined by endothelium; (ii) with supporting smooth muscle or elastica; (iii) filled with lymphatic fluid or red blood cells. Alternative circumstances were considered artifacts due to peritumoral edema and tissue shrinkage.

\section{Statistical analysis}

The Chi-square test was used in order to identify the correlation between LVI and clinicopathologic variables in GC patients. Both DFS and DSS were calculated using the Kaplan-Meier method and the differences between the patient groups were analyzed utilizing a log-rank test in a univariate analysis. A Cox proportional hazard model was utilized for a multivariate analysis in order to determine independent prognostic factors. All tests were two sided and a $P$ value of $<0.05$ was considered to be statistically significant. Statistical analyses were performed using The SPSS 16.0 statistical software (SPSS, Chicago, IL, USA).

\section{Results}

\section{Clinicopathologic characteristics in patients with resectable GC}

The clinicopathological features of our GC cohort are detailed in Table 1. A total of 1148 patients; with a male-to-female ratio of 2.24:1, were included in the present study. The median age at the time of resection was 59.0 years (range, 18.0 to 84.0 years). The presence of LVI was detected in 404 patients (35.2\%); LVI was identified as the invasion of vessel walls by tumor cells (Figure 1A) and/or the presence of tumor emboli within an endothelial-lined space (Figure 1B).

\section{The correlation of LVI with clinicopathologic characteristics in patients with resectable GC}

The correlation between LVI and clinicopathologic characteristics is shown in Table 1. Our analyses support a significant correlation between the presence of LVI and the level of CA19-9, tumor size, Lauren classification, tumor differentiation, infiltration depth, lymph node involvement, distant metastasis and TNM stage $(P=0.004$ for CA19-9 level and Lauren classification; $P<0.0001$

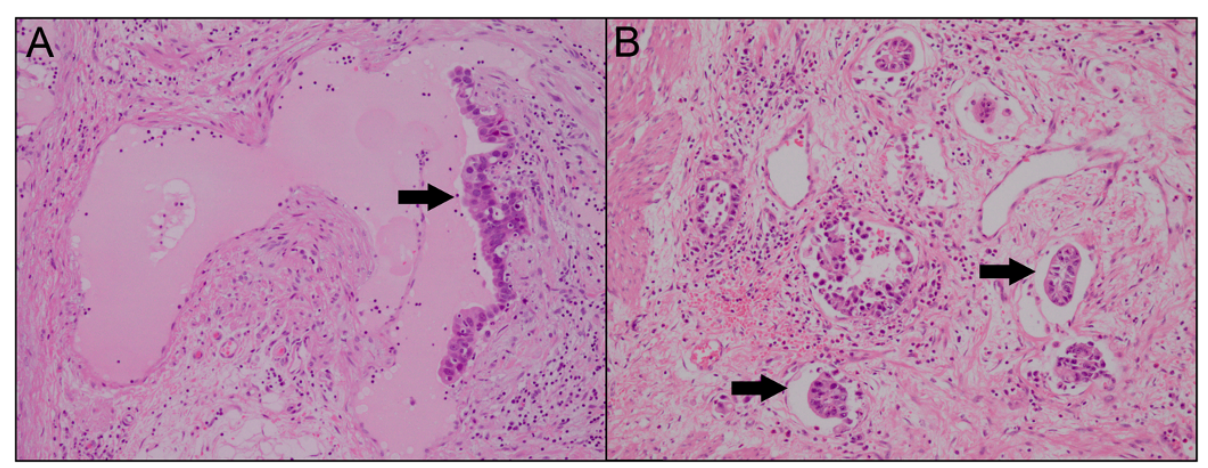

Figure 1 Histological patterns of lymphovascular invasion in gastric cancer. (A) Vessel walls were invaded by tumor cells. (B) Tumor emboli were observed within an endothelium-lined space. 
for the others). However, no significant correlation was found between the presence of LVI and other variables; such as gender, age and CEA level $(P>0.05)$.

\section{The prognostic impact of LVI in patients with resectable GC} The average time interval between surgery and the follow-up examination was 40.4 months (range, 1.0 to 161.5 months). The five-year DSS and DFS rates for all 1148 patients were $51.0 \%$ and $44.6 \%$. The five-year DSS was determined to be $33.1 \%$ in patients with LVI and $60.4 \%$ in patients without LVI; as determined using the log-rank test analysis which also indicated that there was a significant difference between the two groups $(\mathrm{P}<0.0001)$ (Figure 2A) The analysis also indicated that DFS was significantly decreased in patients with LVI as compared to those without LVI $(27.8 \%$ vs. $53.1 \%, P<0.0001)$ (Figure 2B). A stratified analysis was performed to evaluate the correlative impact of identifying LVI at each TNM stage as it relates to patient survival. Our results indicate that the presence of LVI was a reliable prognostic factor for DSS in GC patients with stage I, stage II, stage III or stage IV $(P<0.05$, Figure 3A-D). Similar results were obtained when focusing on DFS. LVI was determined to be a reliable indicator of DFS in stage I or stage III $(P=0.005$ for both) and showed a tendency towards statistical significance when found in stage II $(P=0.086)$ or stage IV $(P=0.067)$, as determined by doing a stage-match survival analysis (Figure 3E-H).

\section{LVI is an independent predictor of poor outcome in patients with resectable GC}

The univariate analysis indicated that certain variables were shown to correlate with DSS; these variables include age at the time of surgery $(P=0.046)$, CA19-9 level $(P=0.001)$, tumor size $(P<0.0001)$, Lauren classification $(P<0.0001)$, tumor differentiation $(P<0.0001)$, infiltration depth $(P<0.0001)$, lymph node metastasis $(P<0.0001)$, distant metastasis $(P<0.0001)$, TNM stage $(P<0.0001)$ and LVI $(P<0.0001$, Table 2$)$. A Cox proportional hazard model was performed using the multivariate analysis in order to determine independent prognostic factors of DSS. The independent variables shown to correlate with the post-surgical DSS were confirmed to be the tumor size (HR, 1.311; 95\%CI, 1.077-1.595, $P=0.007$ ), infiltration depth (HR, 2.284; 95\%CI, 1.413-3.691, $P=0.001$ ), distant metastasis (HR, 2.365; 95\%CI, 1.851-3.022, $P<0.0001$ ), TNM stage (HR, 2.090; 95\%CI, 1.462-2.988, $P<0.0001$ ) and LVI (HR, 1.438; 95\%CI, 1.171-1.766, $P=0.001$ ) (Table 2). Similarly, LVI was found to be an independent prognostic factor for DFS in GC patients after curative resection (HR, 1.393; 95\%CI, 1.150-1.688, $P=0.001$, Table 3).

\section{Discussion}

The presence of LVI, a common pathological finding for a variety of different cancer types, has been of considerable interest in the last few decades as a potential biomarker. The effectiveness of LVI as a reliable indicator of cancer recurrence and prognosis has been clearly established for both hepatocellular carcinoma and testicular cancer, supporting its incorporation into the UICC/AJCC TNM staging system [19,20]. Previous studies have also shown that the presence of LVI correlated with a poor prognosis. However, due to the lack of large, well-designed and prospective studies, at this time LVI is only recommend to be included in final pathological reports rather than being included in the initial TNM staging system of GC as stated in the NCCN Guidelines for Gastric Cancer of 2013 [21].

In this large-scale retrospective study, through the use of $\mathrm{H} \& \mathrm{E}$ staining, LVI was determined to be present in resected GC specimens at a fairly high frequency. Its
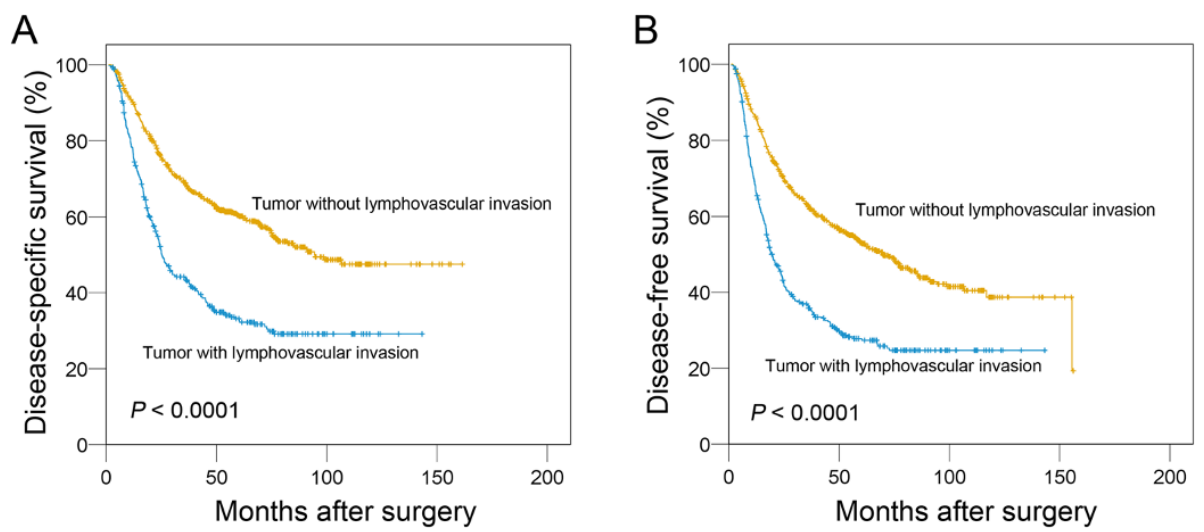

Figure 2 The impact of lymphovascular invasion on the prognosis of patients with gastric cancer (log-rank test). There was a statistically significant difference in the disease-specific survival (A) and disease-free survival (B) between lymphovascular invasion-positive and -negative patients. 

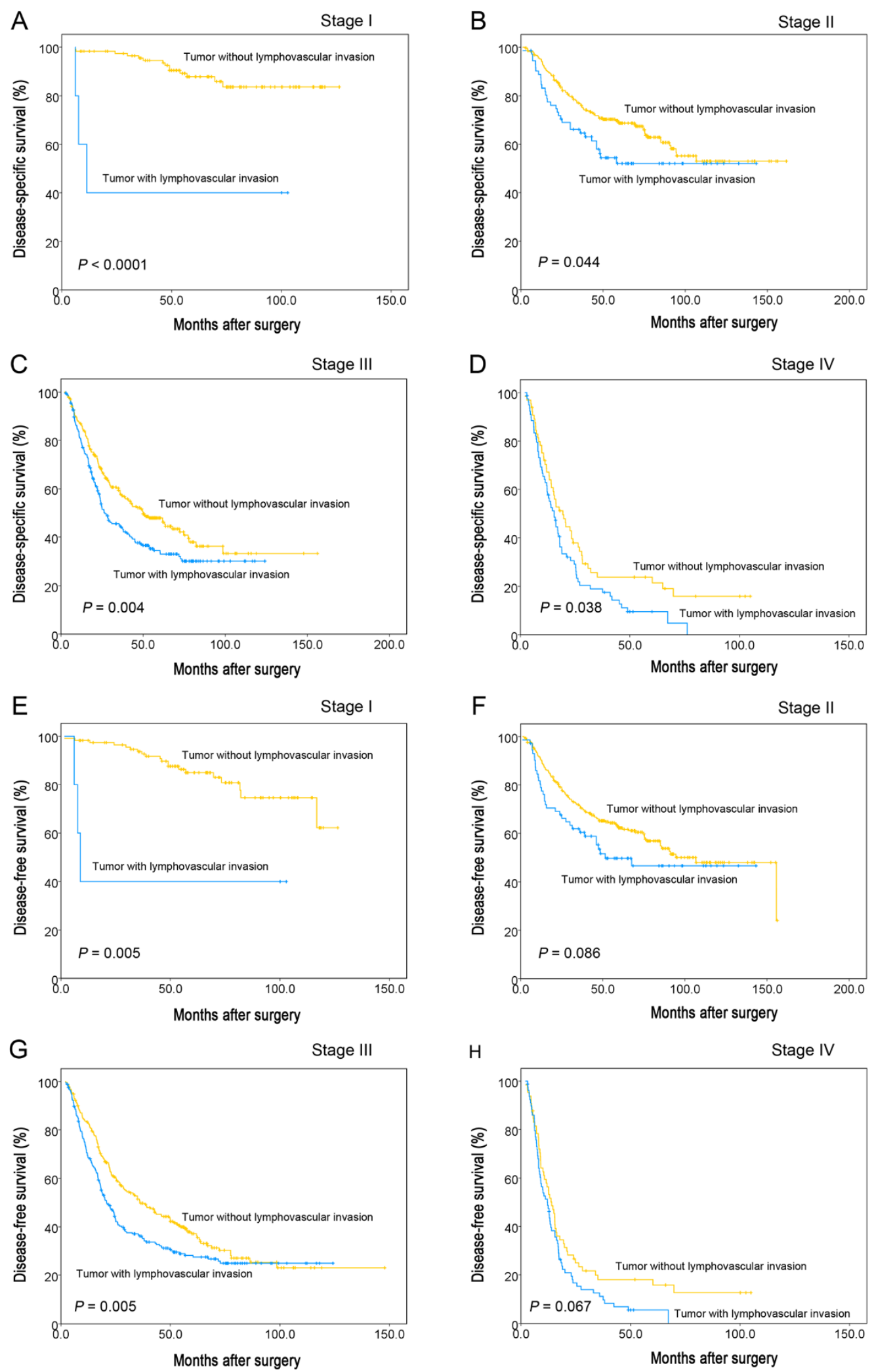

Figure $\mathbf{3}$ (See legend on next page.) 
(See figure on previous page.)

Figure 3 The prognostic significance of lymphovascular invasion in patients stratified by the TNM stage (log-rank test). Stage-match survival analysis showed that the presence of LVI was a prognostic factor for DSS in GC patients with stage I, stage II, stage III or stage IV (A-D). Stage-match survival analysis demonstrated that LVI was a statistically significant predictor for DFS in stage I or stage III and a tendency towards statistical significance was found in stage II $(P=0.086)$ or stage IV $(P=0.067, \mathbf{E}-\mathbf{H})$.

Table 2 Univariate and multivariate analyses of different prognostic factors in 1148 patients with gastric carcinoma for disease-specific survival

\begin{tabular}{|c|c|c|c|c|c|}
\hline \multirow[t]{2}{*}{ Variable } & \multicolumn{3}{|c|}{ Univariate analysis $^{*}$} & \multicolumn{2}{|c|}{ Multivariate analysis } \\
\hline & All cases & HR $(95 \% \mathrm{Cl})$ & $P$ value & $\mathrm{HR}(95 \% \mathrm{Cl})$ & $P$ value \\
\hline Sex & & & 0.800 & & \\
\hline Female & 354 & Reference & & & \\
\hline Male & 794 & 0.977 (0.817-1.169) & & & \\
\hline Age at diagnosis (years) & & & 0.046 & $1.170(0.960-1.425)$ & 0.120 \\
\hline$\leq 59$ & 574 & Reference & & & \\
\hline$>59$ & 574 & $1.184(1.003-1.398)$ & & & \\
\hline $\mathrm{CEA}^{\dagger}$ & & & 0.082 & & \\
\hline Normal & 791 & Reference & & & \\
\hline Elevated & 157 & $1.231(0.974-1.556)$ & & & \\
\hline CA19-9 $9^{\ddagger}$ & & & 0.001 & 1.098 (0.878-1.374) & 0.411 \\
\hline Normal & 707 & Reference & & & \\
\hline Elevated & 195 & 1.438 (1.155-1.791) & & & \\
\hline Size (diameter), cm & & & $<0.0001$ & $1.311(1.077-1.595)$ & 0.007 \\
\hline$\leq 5$ & 697 & Reference & & & \\
\hline$>5$ & 451 & $1.695(1.436-2.000)$ & & & \\
\hline Lauren classification & & & $<0.0001$ & $0.836(0.619-1.129)$ & 0.243 \\
\hline Diffuse & 585 & Reference & & & \\
\hline Mixed/Intestinal & 563 & $0.740(0.627-0.875)$ & & & \\
\hline Differentiation & & & $<0.0001$ & $1.126(0.816-1.554)$ & 0.471 \\
\hline Well/moderate & 435 & Reference & & & \\
\hline Poor/undifferentiated & 713 & $1.445(1.211-1.724)$ & & & \\
\hline Gastric wall invasion & & & $<0.0001$ & $2.284(1.413-3.691)$ & 0.001 \\
\hline $\mathrm{T} 1 / \mathrm{T} 2$ & 150 & Reference & & & \\
\hline $\mathrm{T} 3 / \mathrm{T} 4$ & 998 & $4.643(3.083-6.991)$ & & & \\
\hline Nodal metastasis & & & $<0.0001$ & $0.806(0.573-1.134)$ & 0.216 \\
\hline No & 377 & Reference & & & \\
\hline N1-N3 & 771 & $2.529(2.061-3.102)$ & & & \\
\hline Distant metastasis & & & $<0.0001$ & $2.365(1.851-3.022)$ & $<0.0001$ \\
\hline MO & 1003 & Reference & & & \\
\hline M1 & 145 & $3.479(2.832-4.272)$ & & & \\
\hline TNM stage & & & $<0.0001$ & $2.090(1.462-2.988)$ & $<0.0001$ \\
\hline$|/| \mid$ & 486 & Reference & & & \\
\hline III/ IV & 662 & $3.039(2.516-3.670)$ & & & \\
\hline Vascular invasion & & & $<0.0001$ & 1.438 (1.171-1.766) & 0.001 \\
\hline Absent & 744 & Reference & & & \\
\hline Present & 404 & $2.121(1.795-2.506)$ & & & \\
\hline
\end{tabular}

${ }^{*}$ Cox regression model; ${ }^{\dagger}$ Preoperative serum CEA was measured in 948 patients; ${ }^{\ddagger}$ Preoperative serum CA19-9 was measured in 902 patients; HR indicates hazards ratio; $\mathrm{Cl}$ indicates confidence interval; CEA indicates carcinoembryonic antigen; CA19-9 indicates carbohydrate antigen 19-9. 
Table 3 Univariate and multivariate analyses of different prognostic factors in 1148 patients with gastric carcinoma for disease-free survival

\begin{tabular}{|c|c|c|c|c|c|}
\hline \multirow[t]{2}{*}{ Variable } & \multicolumn{3}{|c|}{ Univariate analysis $^{*}$} & \multicolumn{2}{|c|}{ Multivariate analysis } \\
\hline & All cases & HR $(95 \% \mathrm{Cl})$ & $P$ value & HR $(95 \% \mathrm{Cl})$ & $P$ value \\
\hline Sex & & & 0.936 & & \\
\hline Female & 354 & Reference & & & \\
\hline Male & 794 & 1.007 (0.851-1.192) & & & \\
\hline Age at diagnosis (years) & & & 0.359 & & \\
\hline$\leq 59$ & 574 & Reference & & & \\
\hline$>59$ & 574 & $1.075(0.921-1.255)$ & & & \\
\hline $\mathrm{CEA}^{+}$ & & & 0.068 & & \\
\hline Normal & 791 & Reference & & & \\
\hline Elevated & 157 & $1.227(0.985-1.528)$ & & & \\
\hline CA19-9 $9^{\ddagger}$ & & & $<0.0001$ & $1.145(0.929-1.411)$ & 0.204 \\
\hline Normal & 707 & Reference & & & \\
\hline Elevated & 195 & $1.499(1.221-1.840)$ & & & \\
\hline Size (diameter), cm & & & $<0.0001$ & $1.334(1.111-1.602)$ & 0.002 \\
\hline$\leq 5$ & 697 & Reference & & & \\
\hline$>5$ & 451 & 1.643 (1.407-1.919) & & & \\
\hline Lauren classification & & & 0.001 & $0.821(0.619-1.090)$ & 0.173 \\
\hline Diffuse & 585 & Reference & & & \\
\hline Mixed/Intestinal & 563 & $0.768(0.657-0.897)$ & & & \\
\hline Differentiation & & & $<0.0001$ & $1.085(0.801-1.470)$ & 0.598 \\
\hline Well/moderate & 435 & Reference & & & \\
\hline Poor/undifferentiated & 713 & $1.374(1.166-1.618)$ & & & \\
\hline Gastric wall invasion & & & $<0.0001$ & $2.164(1.416-3.308)$ & $<0.0001$ \\
\hline $\mathrm{T} 1 / \mathrm{T} 2$ & 150 & Reference & & & \\
\hline $\mathrm{T} 3 / \mathrm{T} 4$ & 998 & $4.450(3.083-6.424)$ & & & \\
\hline Nodal metastasis & & & $<0.0001$ & $0.788(0.571-1.088)$ & 0.148 \\
\hline No & 377 & Reference & & & \\
\hline N1-N3 & 771 & $2.545(2.106-3.075)$ & & & \\
\hline Distant metastasis & & & $<0.0001$ & $2.259(1.785-2.858)$ & $<0.0001$ \\
\hline MO & 1003 & Reference & & & \\
\hline M1 & 145 & $3.544(2.906-4.321)$ & & & \\
\hline TNM stage & & & $<0.0001$ & $2.234(1.596-3.127)$ & $<0.0001$ \\
\hline$|/| \mid$ & 486 & Reference & & & \\
\hline III/ IV & 662 & $3.062(2.570-3.649)$ & & & \\
\hline Lymphovascular invasion & & & $<0.0001$ & 1.393 (1.150-1.688) & 0.001 \\
\hline Absent & 744 & Reference & & & \\
\hline Present & 404 & 2.046 (1.749-2.394) & & & \\
\hline
\end{tabular}

${ }^{*}$ Cox regression model; ${ }^{\dagger}$ Preoperative serum CEA was measured in 948 patients; ${ }^{\ddagger}$ Preoperative serum CA19-9 was measured in 902 patients; HR indicates hazards ratio; Cl indicates confidence interval; CEA indicates carcinoembryonic antigen; CA19-9 indicates carbohydrate antigen 19-9.

presence was also shown to correlate with a higher chance of cancer recurrence and was shown to be an independent predictor of a poor survival rate in post-surgical GC patients.
The presence of LVI was detected in $35.2 \%$ of GC patients by $\mathrm{H}$ \& E staining in this study. Similarly, del Casar et al. had previously reported that $31.9 \%$ of GC patients had presented with LVI as detected using $\mathrm{H} \& \mathrm{E}$ 
staining complemented by immunostaining with CD34 [22]. However, a study by Kim et al. had indicated that LVI was detected in $44.3 \%$ of GC patients by immunostaining with D2-40 and CD31 [16]. The differences in the detection rate of LVI could be due to variations in the detection methods. The use of $\mathrm{H}$ \& $\mathrm{E}$ staining, an elastic fiber stain and immunostaining are currently accepted methods in the literature for the detection of LVI. Histological identification of LVI using H \& E staining can be subjective, which could lead to the underestimation of the incidence of LVI. However, successful vessel identification using $\mathrm{H}$ \& $\mathrm{E}$ staining has been previously shown to be sufficiently reliable. With quality control measures in place, the prognostic value of LVI as detected by $\mathrm{H}$ \& $\mathrm{E}$ staining was determined for upper urinary tract urothelial carcinoma, breast cancer, colorectal cancer and non-small cell lung cancer [11,23-25]. Additionally, a previous study indicated that both LVI and BVI, as detected by both $\mathrm{H} \& \mathrm{E}$ and IHC staining, significantly correlated with lymph node metastasis [17]. Consistent with previous findings, our data demonstrates that the presence of LVI, as detected by $\mathrm{H} \& \mathrm{E}$ staining, significantly correlates with DFS and DSS in post-surgical GC patients.

Several small-scale studies have previously noted the prognostic value of LVI on DSS and DFS in GC patients. The presence of LVI was shown to be significantly associated with a poorer OS in 77 patients with primary gastric adenocarcinoma [22]. The three-year OS and threeyear DFS of $149 \mathrm{GC}$ patients were found to be significantly higher in GC patients without LVI as compared to those with LVI [16]. A retrospective analysis indicated that the OS of the LVI-positive patients, out of 436 stage II GC patients, was shown to be worse than that of the LVI-negative patients [26]. Similarly, we confirmed the negative impact of LVI on DSS and DFS in a large cohort of 1148 patients with gastric adenocarcinoma who underwent gastrectomy. Additionally, a stage-stratified survival analysis determined that the presence of LVI in GC patients correlated with a poorer prognostic outcome. Notably, our study identified LVI as an independent prognostic factor through the use of multivariate analysis. Our findings are in agreement with the results of previously published studies $[18,26]$. However, it is of note to point out that LVI was not identified as an independent prognostic factor in GC patients in all of the previous studies identified. Kim et al. had reported that the presence of LVI was shown to have a significant impact on patient survival; however, it was not determined to be an independent prognostic factor in GC. A close relationship between the presence of LVI and tumor progression was speculated to be the basis for this negative result [16].

This study, along with previous reports, supports the view that the presence of LVI in GC is a promising indicator of tumor aggressiveness; providing additional information regarding the risk of cancer recurrence and mortality. The addition of LVI assessment to the current UICC/AJCC TNM staging system may lead to a more accurate risk stratification of affected patients and may lead to more appropriate clinical decision-making. Interestingly, randomized controlled trials have recently demonstrated treatment benefits from adjuvant therapy given to GC patients who have undergone surgery $[4,27,28]$. This supports the idea that GC patients with LVI may be good candidates for further adjuvant therapies that may improve their chances at survival.

The status of nodal metastasis was not evaluated as a statistically significant prognostic factor in multivariate analysis in the present study. However, nodal metastasis was found to be closely correlated with a poor prognosis in our univariate analysis on patient survival. Lymph node status, TNM stage and LVI were included in our multivariate analyses for DSS and DFS. It is known that the status of nodal metastasis is included in TNM staging for $\mathrm{GC}$ and there is a strong association between nodal metastasis status and TNM stage. Meanwhile, in agreement with previously published studies, our data indicate that the status of nodal metastasis significantly correlated with the presence of LVI in GC $[12,22]$. Therefore, the effect of covariate mainly contributes to this negative result.

Consistent with previous studies, the 5-year DSS rate in this study was determined to be approximately $51.0 \%$ for all stages, $70.5 \%$ for stage I-II and $36.1 \%$ for stage IIIIV $[29,30]$. However, the published SEER data indicated that in the United States, the 5-year relative survival for GC was $28.3 \%$ for all stages, $64.1 \%$ for a localized stage, $28.8 \%$ for a regional stage. In fact, it has been suggested that patients with GC have a more favorable prognosis in Asia as compared to those in Europe and the US; a variety of potential reasons have been proposed to explain this. First, the survival advantage of the Asian ethnicity continues to play a role even after being controlled for using other well-known prognostic factors [31,32]. Additionally, the higher surgical quality may contribute to the increased survival rate in Asia; gastrectomy with D2 lymphadenectomy is the standard treatment for GC patients in China. Several clinical trials also have also confirmed the survival benefit for D2 lymph node dissection [33]. Meanwhile, the high incidence of GC in China has subsequently resulted in highly experienced surgeons due to the vast number of times they perform that particular surgery.

Several limitations that could affect the interpretation of our results exist due to the retrospective nature of the study. Potential bias was minimized through the use of strict inclusion and exclusion criteria for patient selection as well as duplicate reviews for each pathologic 
evaluation carried out according to the commonly used unified international criteria. Further validation of our results will require subsequent large-scale prospective studies.

\section{Conclusions}

Routine $\mathrm{H}$ \& E staining to determine LVI could be an effective tool in the identification of GC patients that are at an increased risk of tumor recurrence and/or progression. This could also aid in the selection of the appropriate treatment for each patient depending on their status; such as favoring adjuvant therapies in patients with LVI.

\begin{abstract}
Abbreviations
LVI: Lymphovascular invasion; GC: Gastric cancer; OS: Overall survival; DFS: Disease-free survival; DSS: Disease-specific survival; UICC/AJCC: The International Union Against Cancer/American Joint Committee on Cancer.
\end{abstract}

\section{Competing interests}

The authors declare that they have no competing interests.

\section{Authors' contributions}

MYC is responsible for the study design. PL performed the experiments and drafted the manuscript. HQH carried out the data analysis and interpretation. $\mathrm{CMZ}, \mathrm{YHL}, \mathrm{WMH}, \mathrm{XKZ}, \mathrm{RZL}, \mathrm{JPY}$ and DX participated in the data collection.YFL provided the patients' clinical data. All authors read and approved the final manuscipt.

\section{Acknowledgements}

This study was supported by the grants from the Nature Science Foundation of China (No. 81302139), the Foundation for Distinguished Young Talents in Higher Education of Guangdong (No. 84000-3211701) and the Program for Excellent Young Talents in Sun Yat-sen University Cancer Center (No. 520101210101).

\section{Author details \\ ${ }^{1}$ State Key Laboratory of Oncology in South China, Collaborative Innovation Center for Cancer Medicine, Sun Yat-sen University Cancer Center, Guangzhou, China. ${ }^{2}$ Department of Pathology, Sun Yat-sen University Cancer Center, No. 651, Dongfeng Road East, 510060 Guangzhou, China. ${ }^{3}$ Diagnostic Imaging and Intervening Center, Sun Yat-sen University Cancer Center, Guangzhou, China. ${ }^{4}$ Department of Gastric \& Pancreatic Surgery, Sun Yat-sen University Cancer Center, No. 651, Dongfeng Road East, 510060 Guangzhou, China.}

Received: 30 November 2014 Accepted: 25 April 2015

Published online: 07 May 2015

\section{References}

1. Desai AM, Pareek M, Nightingale PG, Fielding JW. Improving outcomes in gastric cancer over 20 years. Gastric Cancer. 2004;7(4):196-201. discussion 201-203.

2. Rahman R, Asombang AW, Ibdah JA. Characteristics of gastric cancer in Asia. World J Gastroenterol. 2014;20(16):4483-90.

3. Cuschieri A, Weeden S, Fielding J, Bancewicz J, Craven J, Joypaul V, et al. Patient survival after D1 and D2 resections for gastric cancer: long-term results of the MRC randomized surgical trial. Br J Cancer. 1999;79(9-10):1522-30.

4. Cunningham D, Allum WH, Stenning SP, Thompson JN, Van de Velde CJ, Nicolson $\mathrm{M}$, et al. Perioperative chemotherapy versus surgery alone for resectable gastroesophageal cancer. N Engl J Med. 2006;355(1):11-20.

5. Zhang J, Zhou Y, Jiang K, Shen Z, Ye Y, Wang S. Evaluation of the seventh AJCC TNM staging system for gastric cancer: a meta-analysis of cohort studies. Tumour Biol. 2014;35(9):8525-32.

6. Mohammed RA, Martin SG, Gill MS, Green AR, Paish EC, Ellis IO. Improved methods of detection of lymphovascular invasion demonstrate that it is the predominant method of vascular invasion in breast cancer and has important clinical consequences. Am J Surg Pathol. 2007;31(12):1825-33.
7. Zlobec I, Baker K, Minoo P, Jass JR, Terracciano L, Lugli A. Node-negative colorectal cancer at high risk of distant metastasis identified by combined analysis of lymph node status, vascular invasion, and Raf-1 kinase inhibitor protein expression. Clin Cancer Res. 2008;14(1):143-8.

8. Mete O, Asa SL. Pathological definition and clinical significance of vascular invasion in thyroid carcinomas of follicular epithelial derivation. Mod Pathol. 2011;24(12):1545-52.

9. Maeda R, Yoshida J, Ishii G, Hishida T, Nishimura M, Nagai K. Prognostic impact of intratumoral vascular invasion in non-small cell lung cancer patients. Thorax. 2010;65(12):1092-8.

10. Pichler M, Hutterer GC, Chromecki TF, Jesche J, Groselj-Strele A, Kampel-Kettner K, et al. Prognostic value of the Leibovich prognosis score supplemented by vascular invasion for clear cell renal cell carcinoma. J Urol. 2012;187(3):834-9.

11. Kikuchi E, Margulis V, Karakiewicz PI, Roscigno M, Mikami S, Lotan Y, et al. Lymphovascular invasion predicts clinical outcomes in patients with node-negative upper tract urothelial carcinoma. J Clin Oncol. 2009;27(4):612-8.

12. Bu Z, Zheng Z, Li Z, Zhang L, Wu A, Wu X, et al. Lymphatic vascular invasion is an independent correlated factor for lymph node metastasis and the prognosis of resectable T2 gastric cancer patients. Tumour Biol. 2013;34(2):1005-12.

13. Setala LP, Kosma VM, Marin S, Lipponen PK, Eskelinen MJ, Syrjanen KJ et al. Prognostic factors in gastric cancer: the value of vascular invasion, mitotic rate and lymphoplasmacytic infiltration. Br J Cancer. 1996;74(5):766-72.

14. Maehara Y, Kabashima A, Koga T, Tokunaga E, Takeuchi H, Kakeji Y, et al. Vascular invasion and potential for tumor angiogenesis and metastasis in gastric carcinoma. Surgery. 2000;128(3):408-16.

15. Cao F, Hu YW, Li P, Liu Y, Wang K, Ma L, et al. Lymphangiogenic and angiogenic microvessel density in chinese patients with gastric carcinoma: correlation with clinicopathologic parameters and prognosis. Asian Pac J Cancer Prev. 2013;14(8):4549-52.

16. Kim JH, Park SS, Park SH, Kim SJ, Mok YJ, Kim CS, et al. Clinical significance of immunohistochemically-identified lymphatic and/or blood vessel tumor invasion in gastric cancer. J Surg Res. 2010;162(2):177-83.

17. Gresta LT, Rodrigues-Junior IA, de Castro LP, Cassali GD, Cabral MM. Assessment of vascular invasion in gastric cancer: a comparative study. World J Gastroenterol. 2013;19(24):3761-9.

18. Gabbert HE, Meier S, Gerharz CD, Hommel G. Incidence and prognostic significance of vascular invasion in 529 gastric-cancer patients. Int J Cancer. 1991;49(2):203-7.

19. Vauthey JN, Lauwers GY, Esnaola NF, Do KA, Belghiti J, Mirza N, et al. Simplified staging for hepatocellular carcinoma. J Clin Oncol. 2002;20(6):1527-36.

20. Albers P, Siener R, Kliesch S, Weissbach L, Krege S, Sparwasser C, et al. Risk factors for relapse in clinical stage I nonseminomatous testicular germ cell tumors: results of the German Testicular Cancer Study Group Trial. J Clin Oncol. 2003;21(8):1505-12.

21. NCCN. Clinical Practice Guidelines in Oncology: Gastric Cancer, Version 2. 2013. National Comprehensive Cancer Network, Inc. http://www.nccn.org/ professionals/physician_gls/pdf/gastric.pdf. Accessed July 23, 2013.

22. del Casar JM, Corte MD, Alvarez A, Garcia I, Bongera M, Gonzalez LO, et al. Lymphatic and/or blood vessel invasion in gastric cancer: relationship with clinicopathological parameters, biological factors and prognostic significance. J Cancer Res Clin Oncol. 2008;134(2):153-61.

23. Lee AH, Pinder SE, Macmillan RD, Mitchell M, Ellis IO, Elston CW, et al. Prognostic value of lymphovascular invasion in women with lymph node negative invasive breast carcinoma. Eur J Cancer. 2006;42(3):357-62.

24. Betge J, Pollheimer MJ, Lindtner RA, Kornprat P, Schlemmer A, Rehak $P$, et al. Intramural and extramural vascular invasion in colorectal cancer: prognostic significance and quality of pathology reporting. Cancer. 2012;118(3):628-38.

25. Ruffini E, Asioli S, Filosso PL, Buffoni L, Bruna MC, Mossetti C, et al. Significance of the presence of microscopic vascular invasion after complete resection of Stage I-II pT1-T2NO non-small cell lung cancer and its relation with T-Size categories: did the 2009 7th edition of the TNM staging system miss something? J Thorac Oncol. 2011;6(2):319-26.

26. Du CY, Chen JG, Zhou Y, Zhao GF, Fu H, Zhou XK, et al. Impact of lymphatic and/or blood vessel invasion in stage II gastric cancer. World J Gastroenterol. 2012;18(27):3610-6.

27. Walsh TN, Noonan N, Hollywood D, Kelly A, Keeling N, Hennessy TP. A comparison of multimodal therapy and surgery for esophageal adenocarcinoma. N Engl J Med. 1996;335(7):462-7. 
28. Bang YJ, Kim YW, Yang HK, Chung HC, Park YK, Lee KH, et al. Adjuvant capecitabine and oxaliplatin for gastric cancer after D2 gastrectomy (CLASSIC): a phase 3 open-label, randomised controlled trial. Lancet. 2012;379(9813):315-21.

29. Lu J, Huang CM, Zheng CH, Li P, Xie JW, Wang JB, et al. Analysis on the clinical and pathological features and prognosis of familial gastric cancer in South china population: a single-center study of 724 patients. J Oncol. 2012;2012:641218.

30. Zheng L, Wu C, Xi P, Zhu M, Zhang L, Chen $S$, et al. The survival and the long-term trends of patients with gastric cancer in Shanghai, China. BMC Cancer. 2014;14:300.

31. Wang J, Sun Y, Bertagnolli MM. Comparison of gastric cancer survival between caucasian and Asian patients treated in the United States: results from the Surveillance Epidemiology and End Results (SEER) Database. Ann Surg Oncol. 2015. [Epub ahead of print].

32. Lui FH, Tuan B, Swenson SL, Wong RJ. Ethnic disparities in gastric cancer incidence and survival in the USA: an updated analysis of 1992-2009 SEER data. Dig Dis Sci. 2014;59(12):3027-34.

33. Songun I, Putter H, Kranenbarg EM, Sasako M, van de Velde CJ. Surgical treatment of gastric cancer: 15-year follow-up results of the randomised nationwide Dutch D1D2 trial. Lancet Oncol. 2010;11(5):439-49.

\section{Submit your next manuscript to BioMed Central and take full advantage of:}

- Convenient online submission

- Thorough peer review

- No space constraints or color figure charges

- Immediate publication on acceptance

- Inclusion in PubMed, CAS, Scopus and Google Scholar

- Research which is freely available for redistribution 